\title{
Reduction of physical activity during the COVID-19 pandemic is related to increased neuropsychiatric symptoms in memory clinic patients
}

\author{
Authors: Raphael Wurm, ${ }^{\mathrm{A}}$ Tandis Parvizi, ${ }^{\mathrm{A}}$ Sara Silvaeih, ${ }^{\mathrm{A}}$ Evelyn Berger-Sieczkowski, ${ }^{\mathrm{B}}$ Stella Goeschl, ${ }^{\mathrm{C}}$ \\ Theresa König, Johann Lehrner ${ }^{\mathrm{E}}$ and Elisabeth Stögmann ${ }^{\mathrm{D}}$
}

The COVID-19 pandemic led to unprecedented restrictions on social contacts and mobility. Memory clinic patients were disproportionately affected when care was disrupted and routines were abruptly changed. This trial was designed as a pragmatic, prospective, observational study to evaluate the effects of lockdown on memory clinic patients. Outpatients were included when they returned in May to July 2020 for their first follow-up after the lockdown. Indicators of lockdown intensity and its effect on patients were recorded, patients and caregivers were interviewed, and neuropsychological tests were performed. We included 72 patients, most of them suffering from Alzheimer's dementia or mild cognitive impairment. The median time of isolation was 8 weeks and social contacts were significantly reduced from five to two per week $(p<0.001)$. Light physical activity was significantly reduced ( 3.8 hours to 3 hours, $p=0.016$ ) during the lockdown, and this reduction was significantly correlated with higher scores on the Neuropsychiatric Inventory score ( $\mathrm{-}-0.43, \mathrm{p}>0.001)$. Fears regarding the pandemic were common and mostly related to the patients' health. Lockdown restrictions reduced physical activity in memory clinic patients which was associated with increased neuropsychiatric symptoms. Future restrictions should aim to mitigate the impacts on this vulnerable population.

KEYWORDS: COVID-19, dementia, neuropsychiatric symptoms, physical activity

DOI: 10.7861/clinmed.2021-0605

Authors: Aneurology registrar, Medical University of Vienna, Vienna, Austria; ${ }^{B}$ consultant in neurology, Medical University of Vienna, Vienna, Austria; ' medical student, Medical University of Vienna, Vienna, Austria; Ddoctoral candidate, Medical University of Vienna, Vienna, Austria; Eprofessor of clinical psychology, Medical University of Vienna, Vienna, Austria; Fprofessor of neurology, Medical University of Vienna, Vienna, Austria.

\section{Introduction}

The COVID-19 pandemic led to unprecedented restrictions across the globe. On 17 March 2020, Austria announced its first lockdown, effectively confining its residents to their homes except for commuting, strictly necessary errands, helping others or physical activity. ${ }^{1}$ This greatly reduced social contacts and led to a dramatic fall in mobility patterns across the country. ${ }^{2}$ Restrictions were gradually eased in April and May 2020. While they proved highly effective in suppressing the first wave of the pandemic, social distancing and isolation had a pronounced negative impact on psychological wellbeing in the general population. ${ }^{3}$ Patients with dementia were disproportionately affected, owing in part to pre-existing disease-related neuropsychiatric symptoms and to the abrupt discontinuation of institutionalised care services such as day-care centres, therapists and 24-hour care. ${ }^{4}$ Symptoms of anxiety, apathy and agitation worsened in one cohort of Spanish patients with dementia, ${ }^{5}$ while deterioration was more prominent in patients with lower baseline cognitive function in another study. ${ }^{6}$ With the prospect of repeated constraints due to the COVID-19 pandemic, tailoring future interventions to minimise the negative impact on the especially vulnerable group of patients with dementia is paramount and should incorporate regional differences and experiences. ${ }^{7}$ This study was conceived out of the necessity to better understand the effects of the pandemic on our population and designed as a pragmatic, exploratory analysis.

\section{Methods}

This study was designed as a pragmatic, prospective, single-centre observational trial conducted in the memory clinic of a tertiary care centre. We included all patients that returned for their first follow-up visit after lockdown restrictions were eased and outpatient clinics were reopened in Vienna between May and July 2020.

Patients and caregivers were asked to recall a typical week prior to the pandemic, ie in February 2020, and a typical week during lockdown. We recorded the duration of strict social isolation, as some patients chose to self-quarantine for longer durations. Patients were asked to recall the average number of individual social contacts prior to and during the pandemic, and to give the cumulative 


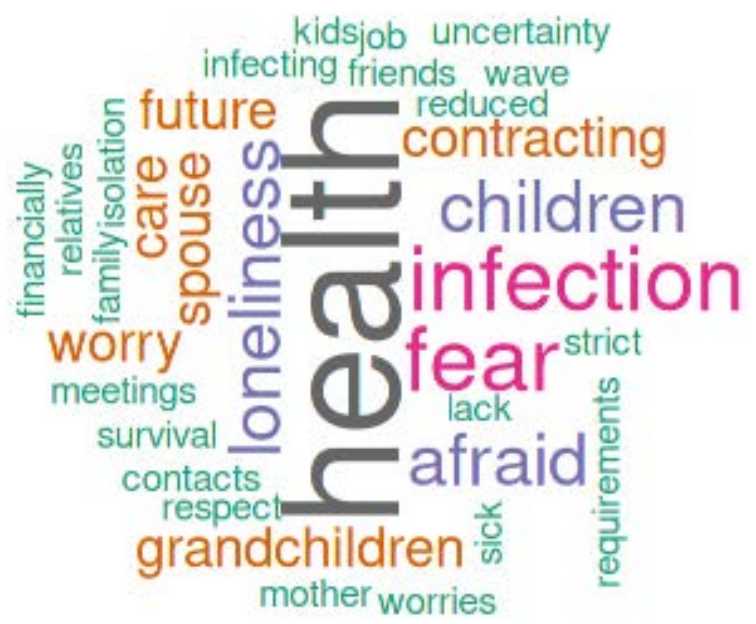

Fig 1. Word cloud depicting leading sentiments of memory clinic patients when asked about their fears or worries with regards to the COVID-19 pandemic. More frequent words appear larger.

duration of physical activities. They were then grouped into vigorous (eg weight lifting, high-intensity cycling, running), moderate (eg nordic walking, light cycling) and light (eg non-necessary walks, walking a pet) and recorded as hours per week.

For the qualitative part, patients were asked to reflect on their fears and worries with regards to the pandemic and state them in one sentence. The collected sentences were translated, stop words such as ' $a$ ' and 'the' were removed, and the resulting expressions were visualised in a word cloud with words appearing larger by frequency, using the R package wordcloud. ${ }^{8}$ Additionally, they were then categorised into the leading sentiment. We also asked if and how the pandemic and associated restrictions had influenced quality of care. The Mini Mental State Examination (MMSE) test was performed and the Neuropsychiatric Inventory (NPI) was conducted by asking caregivers to recall symptoms during the lockdown. ${ }^{9}$

Numbers are reported as $\mathrm{n}(\%)$, mean (+/- standard deviation) or median (interquartile range, IQR) as appropriate. Differences in contacts or physical activity prior to and during the pandemic were assessed using Wilcoxon's signed-rank test and correlations were assessed using Spearman's rho.

Statistical testing was performed using R (4.0.4, R Core Team (2021). Vienna, Austria). The study was approved by the institutional review board of the Medical University of Vienna (1323/2018).

\section{Results}

\section{Patients}

We included 72 consecutive patients visiting our dementia outpatient clinic for their first regular follow up visit after quarantine restrictions were eased in Austria. Of these patients $44 \%$ were female and the median age at visit was 72.5 years (IQR 64.7579.25). Most patients were diagnosed with Alzheimer's dementia $(44 \%)$ or mild cognitive impairment (30\%), while $10 \%$ suffered from rare forms (such as dementia with Lewy bodies, frontotemporal dementia or corticobasal degeneration) and $16 \%$ were classified as having unspecified dementia. The median duration of disease was 2.3 years (IQR 1-5.1) and patients scored a median of 22.5 points (IQR 17.3-26.8) on the MMSE.

Patients had been isolated, ie their instances of social contacts had been severely reduced and their movement restricted, for a median of 8 weeks (IQR 8-10).

\section{Isolation, lockdown effects on dementia care and fears}

The median number of individual social contacts in an average week prior to the pandemic was five (IQR 4-8) which was significantly reduced to a median of two (IQR $1-4, p<0.001) .16$ patients $(22 \%$ ) compensated for this by using digital technologies, eg video calls, during the lockdown. The restrictions negatively affected care for 11 patients ( $15 \%$ ), due to eg stay-in nurses not being able to enter the country, or to clubs for senior citizens, churches and therapists' offices being closed. When asked about their fears or worries regarding the pandemic, 29 patients ( $42 \%$ ) reported none. The most frequently stated concerns related to the patient's own health $(34 \%)$ or that of their immediate relatives $(23 \%)$, but fears regarding their financial or professional future $(13 \%)$, isolation or loneliness (17\%) or another lockdown (3\%) were also voiced (Fig 1).

\section{Physical activity and neuropsychiatric symptoms}

Very low levels of vigorous and moderate physical activity prior to the pandemic were reported (medians of 0 hours, means of 0.15 and 0.38 hours, respectively) and there was no significant change during the lockdown. The hours of light activity per week were reduced significantly during the lockdown from a median of 3.8 (IQR $1.4-7)$ to $3(1-4.6)$ hours per week ( $p=0.016)$. The NPI score ranged from 0 to 75 with a mean of 11.1 (standard deviation +/- 14.6) points. 12 patients $(17 \%)$ were completely unimpaired, ie NPI of 0 , and $41(57 \%)$ were clinically affected, ie a score of $\geq 4$ in at least one domain. The most frequently affected domains were depression in $23(32 \%)$, apathy in $13(18 \%)$, fear in $9(13 \%)$ and disturbed nighttime behaviour in $8(11 \%)$ patients. All other subdomains were clinically affected in $5(7 \%)$ or less patients. Higher NPI scores were significantly correlated with a larger decrease in light activity during lockdown compared to before $(R=-0.43, p<0.001$, Fig 2$)$, but there was no correlation with MMSE.

\section{Discussion}

We present quantitative and qualitative data showing that lockdown restrictions led to reduced mobility and physical activity in patients with dementia. These reductions were associated with increased neuropsychiatric symptoms. These data add to the growing literature regarding this especially vulnerable population and should inform policy making and mitigation strategies. Data were collected on patients with dementia from a pragmatic, prospective sample treated at an urban university hospital. Data were available on two points in time to assess changes and their influence on neuropsychiatric symptoms.

Our patients significantly reduced their social contacts during lockdown to a median of two individuals per week, including their partners within the same household. Self-reported levels of physical activity were generally low in our sample and light activity was further significantly reduced during the lockdown. This is comparable to patients with dementia at normal times ${ }^{10}$ and the reductions of activity seen in the general population. ${ }^{11}$ Assessing neuropsychiatric 


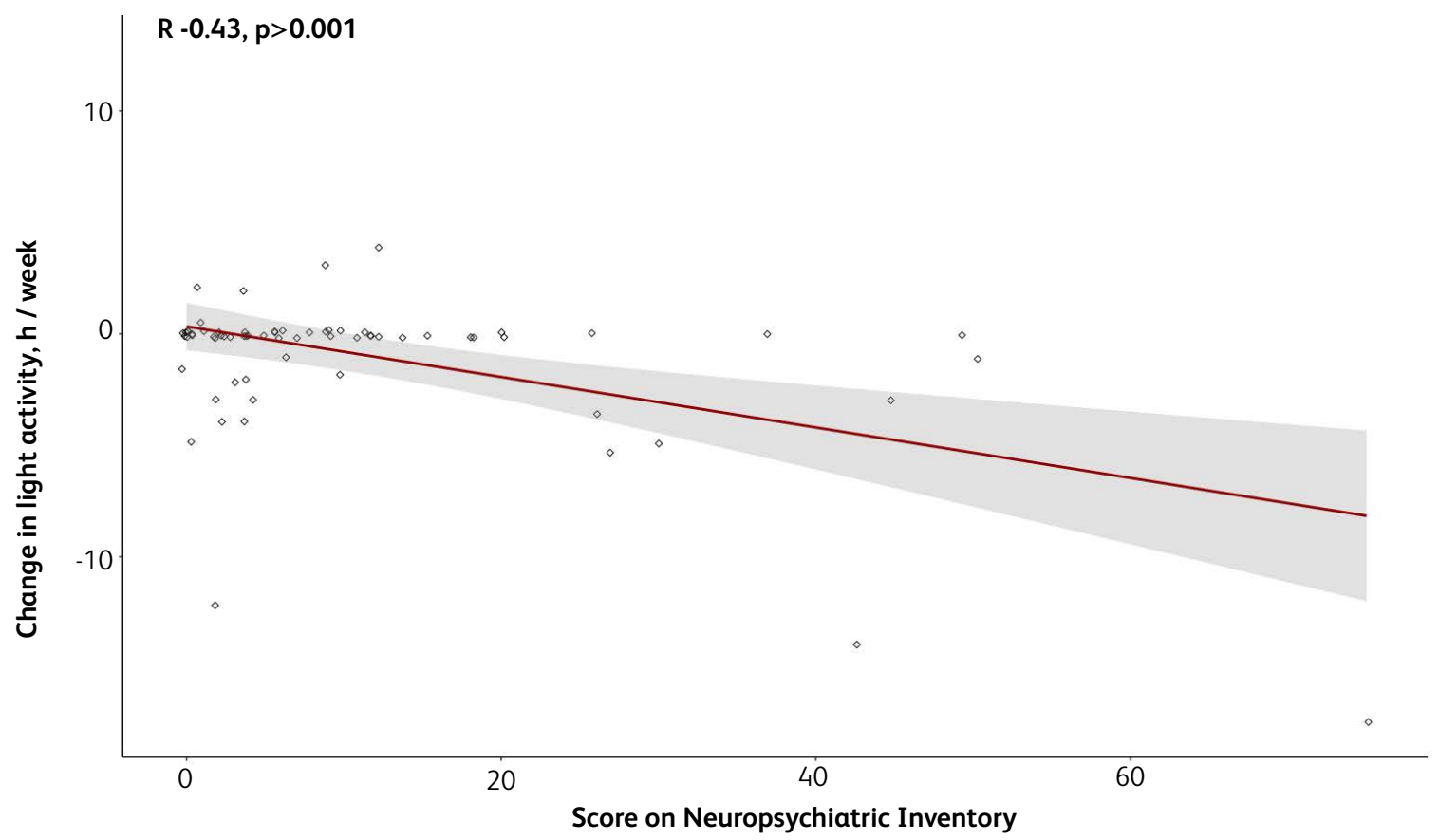

Fig 2. Scatterplot depicting the change in light activity during a COVID-19 lockdown and the total score on the Neuropsychiatric Inventory scale.

symptoms, we found a high rate of clinical impact with depression, apathy and fear being most common. Higher reductions in light activity were significantly correlated with a higher overall score on the NPI. Physical activity is recommended to prevent dementia and other age-related diseases, but it has also been shown to be effective in the management of neuropsychiatric symptoms. ${ }^{12}$ An association of physical activity with neuropsychiatric symptoms thus appears plausible and people with dementia would likely benefit from home-based, remotely instructed forms of exercise. ${ }^{13}$ We found, however, that few of our patients were using digital communication tools.

$15 \%$ reported that the lockdown impacted their dementia-specific care when stay-in nurses could not enter the country or therapists' offices were closed without replacement. At the same time, the mandated closure of clubs for senior citizens, day-care centres and other places of gathering for patients with dementia abruptly changed the structure of a typical day. This can be especially impactful in a population where the adherence to a structured daily routine has been shown to be beneficial, ${ }^{14}$ and a lack thereof could contribute to the neuropsychiatric symptoms in our cohort.

Fears or worries regarding the pandemic were common and mostly related to the patients' health or that of their relatives; however, increased loneliness and more general worries about an uncertain future were also prevalent, as is seen in the wider population during the pandemic. ${ }^{15}$

This study is limited by its retrospective design in that patients and caregivers were asked to recall facts or symptoms in the past. This is of course complicated in patients with memory deficits, but most patients included in this trial were affected mildly. However, recall bias cannot be excluded in patients and caregivers.

Our results highlight the specific needs of people with dementia during this pandemic. While immunisation is rolling out around the world, it becomes obvious that (localised) restrictions will remain part of our response. Further developments of digital solutions for the care of the elderly along with more emphasis on traditional forms of communication should be used to increase physical activity and facilitate social interaction. This could reduce neuropsychiatric symptoms and future regulations should actively provide means of mitigation by timely communication and allowing for patients and caregivers to restructure their days.

\section{References}

1 Federal Chancellery of the Republic of Austria. Verordnung gemäß $\S 2 Z 1$ des COVID-19-Maßnahmengesetzes. Federal Chancellery of the Republic of Austria, 2020. www.ris.bka.gv.at/Dokumente/ BgblAuth/BGBLA_2020_II_98/BGBLA_2020_II_98.pdfsig [Accessed 15 March 2021].

2 Complexity Science Hub Vienna. Different lockdowns, different mobility in Austria. CSH, 2021. www.csh.ac.at/lockdowns-andmobility-in-austria/ [Accessed 15 March 2021].

3 Benke C, Autenrieth LK, Asselmann E, Pané-Farré CA. Lockdown, quarantine measures, and social distancing: Associations with depression, anxiety and distress at the beginning of the COVID-19 pandemic among adults from Germany. Psychiatry Res 2020;293:113462.

4 Mok VCT, Pendlebury S, Wong A et al. Tackling challenges in care of Alzheimer's disease and other dementias amid the COVID-19 pandemic, now and in the future. Alzheimers Dement 2020;16:1571-81.

5 Lara B, Carnes A, Dakterzada F, Benitez I, Piñol-Ripoll G. Neuropsychiatric symptoms and quality of life in Spanish patients with Alzheimer's disease during the COVID-19 lockdown. Eur ] Neurol 2020;27:1744-7.

6 Boutoleau-Bretonnière C, Pouclet-Courtemanche H, Gillet A et al. The effects of confinement on neuropsychiatric symptoms in Alzheimer's disease during the COVID-19 crisis. J Alzheimers Dis 2020;76:41-47.

7 Brown EE, Kumar S, Rajji TK, Pollock BG, Mulsant BH. Anticipating and mitigating the impact of the COVID-19 pandemic on Alzheimer's disease and related dementias. Am J Geriatr Psychiatry 2020;28:712-21.

8 Fellows I. wordcloud: Word clouds. https://CRAN.R-project.org/ package =wordcloud [Accessed 15 March 2021].

9 Cummings JL, Mega M, Gray K et al. The Neuropsychiatric Inventory: 
comprehensive assessment of psychopathology in dementia. Neurology 1994;44:2308-14.

10 Hartman YAW, Karssemeijer EGA, van Diepen LAM, Olde Rikkert MGM, Thijssen DHJ. Dementia patients are more sedentary and less physically active than age- and sex-matched cognitively healthy older adults. Dement Geriatr Cogn Disord 2018;46:81-9.

11 Ammar A, Trabelsi K, Brach M et al. Effects of home confinement on mental health and lifestyle behaviours during the COVID-19 outbreak: Insight from the ECLB-COVID19 multicenter study. Biol Sport 2021:38:9-21.

12 Kouloutbani K, Venetsanou F, Markati A, Karteroliotis KE, Politis A. The effectiveness of physical exercise interventions in the management of neuropsychiatric symptoms in dementia patients: a systematic review. Int Psychogeriatr 2021;1-14.

13 Chen P, Mao L, Nassis GP, Harmer P, Ainsworth BE, Li F. Coronavirus disease (COVID-19): The need to maintain regular physical activity while taking precautions. J Sport Health Sci 2020;9:103-104.
14 Fleiner T, Dauth H, Zijlstra W, Haussermann P. A structured physical exercise program reduces professional caregiver's burden caused by neuropsychiatric symptoms in acute dementia care: randomized controlled trial results. J Alzheimers Dis 2020;74:429-33.

15 Coelho CM, Suttiwan P, Arato N, Zsido AN. On the nature of fear and anxiety triggered by COVID-19. Front Psychol 2020;11:581314.

Address for correspondence: Assoc Prof Dr Elisabeth Stögmann, Univ. Klinik für Neurologie, Währinger Gürtel 18-20, 1090 Wien, Austria.

Email: elisabeth.stoegmann@meduniwien.ac.at 